\title{
Abuse liability—study the intended patient populations
}

Alec B. O'Connor, Dennis C. Turk and Robert H. Dworkin

Eija Kalso and Kaarlo Simojoki object to one of the recent IMMPACT recommendations, which they imply is unethical (Kalso, E. A. \& Simojoki, K. Measuring abuse liabilityis the risk worth taking? Nat. Rev. Neurol. 10, 131-133; 2014). ${ }^{1}$ Our specific recommendation was "the study sample should be chosen to represent the range of possible patients who will be prescribed the drug in practice" in clinical trials intended to assess potential abuse liability (see Box 1 for full recommendations; $2 \mathrm{~b}$ is the most relevant). ${ }^{2}$ Kalso and Simojoki argue that the efficacy of long-term opioid use is not established and, consequently, inclusion of high-risk patients in clinical trials is unethical. The IMMPACT recommendations did not attempt to define the intended populations for specific analgesics with abuse potential. We agree that there are patients for whom the risks of an analgesic clearly outweigh the anticipated benefits, and that these patients should not receive the analgesic in clinical practice or in a clinical trial. It goes without saying that any clinical trial must be built on clinical equipoise, and that all study participants must be given proper protection and treatment, including appropriate psychiatric care and multidisciplinary care, when indicated.

Kalso and Simojoki seem to argue that individuals with more than minimal risk of aberrant drug-taking behaviour should be excluded from clinical trials in which one of the objectives is to assess abuse potential. The science of predicting which patients will do poorly with medications with abuse potential is not well developed. As Kalso and Simojoki acknowledge, studies have identified a number of factors associated with aberrant drug-taking behaviours, including alcoholism, smoking, psychiatric disorders, and family history of addiction. Clinical trials involving analgesics with abuse potential have often excluded patients with such risk factors, although many such patients are treated in practice. Systematically excluding all patients with, for example, psychiatric comorbidity or a family history of addiction from analgesic clinical trials in which assessment of abuse potential is an objective would make it difficult to adequately evaluate the risks of such treatments. In our view, it is essential that a balanced estimate of the risks and benefits of a drug be obtained before the drug is released onto the market. Clinical trials that exclude all but the lowestrisk patients may underestimate the true risk of the drug to patients in the community.

Kalso and Simojoki argue that epidemiological surveillance studies can clarify whether a drug carries increased risk in certain populations that receive the drug, and these studies can then inform post-marketing regulation. Although epidemiological studies certainly have a role in monitoring of abuse liability following initial approval, they require many thousands of patients to be exposed to a drug, it is often unclear whether a true signal is present, and causality cannot be determined from any identified associations.

Box 1 Recommended research design features of clinical trials for assessing abuse liability

1. Characterization of baseline risk of the study sample:

a. Reporting the presence of:

i. Prior opioid abuse

ii. Prior alcohol and/or substance abuse

iii. Family history of opioid, other drug, and alcohol abuse

iv. Smoking history

v. Depression and other psychiatric disorders

b. Urine drug testing (UDT).

c. Consideration of the use of a self-administered tool, such as the SOAPP-R. ${ }^{3}$

2. Relevant trial design features:

a. Certificate of Confidentiality should be obtained before study start in order to protect subject's data. Subjects should be informed that their data is confidential and will be protected from forced disclosure.

b. The study sample should be chosen to represent the range of possible patients who will be prescribed the drug in practice. For example, individuals with greater than minimal baseline risk of aberrant drug-related behaviours (ADRB) should be included in at least some trials being conducted for the evaluation of an analgesic drug with potential abuse liability, and pain patients with psychiatric comorbidities should not be excluded based solely on having psychiatric disease.

c. Consideration should be given to designing some later-stage trials with adequate power to detect minimally important treatment group differences for abuse-related primary or secondary outcome measures. When abuse-related primary outcome measures are used, secondary outcome measures should include measurement of pain-related outcomes. Such trials have the potential to provide a stronger evidence base for estimating the abuse potential of a drug in the patients for whom it will be prescribed.

d. Ideally a trial would include comparators that are both positive (e.g. an existing opioid) and negative (e.g. an analgesic without abuse potential) controls, though this will not always be possible.

3. Outcome measurement domains:

a. Observations by trained study personnel for signs suggestive of abuse and other evidence of ADRB.

b. Use of a structured adverse events query form to include specific questions about the presence of certain symptoms, including euphoria, craving, feeling drunk, hallucinations, overdose, and withdrawal symptoms.

c. UDT results, both for baseline population risk assessment and for monitoring during the course of the trial. Ideally, UDT for the study drug would be followed and documented.

d. Monitoring for evidence that subjects abused or diverted opioids, including reports of running out of study medication early, repeated accidental damage to study medication, or lost or stolen study medication.

e. The use of instruments designed to detect ADRB (e.g. the $\mathrm{COMM}^{4}$ ).

f. Formal screening of family members or domestic partners for evidence of abuse or diversion by subjects.

g. Careful description and analysis of the reasons that subjects terminated trial participation prematurely.

Permission obtained from the International Association for the Study of Pain ${ }^{\circledast}$ (IASP). ๑ 0'Connor, A. B. et al., Pain 154, 2324-2334 (2013). 


\section{CORRESPONDENCE}

Within a clinical trial, all participants provide informed consent and are closely monitored. Exposing large numbers of patients in the community to analgesics without informing them about the risks and expecting that epidemiological studies will eventually provide clarity could result in unforeseen harm, as the history of opioid prescribing during the past 20 years suggests.

Department of Medicine, University of Rochester School of Medicine and Dentistry, 601 Elmwood Avenue, Box HMD, Rochester, NY 14642, USA
(A.B.O.). Department of Anaesthesiology \& Pain Medicine, University of Washington, Box 356540 , Seattle, WA 98195, USA (D.C.T.). Departments of Anaesthesiology, Neurology, Oncology, and Psychiatry, University of Rochester School of Medicine and Dentistry, 601 Elmwood Avenue, Box 604, Rochester, NY 14642, USA (R.H.D.). Correspondence to: D.C.T

turkdc@uw.edu

Competing interests

The authors declare no competing interests.

1. Kalso, E. A. \& Simojoki, K. Measuring abuse liability-is the risk worth taking? Nat. Rev. Neurol. 10, 131-133 (2014).
2. O'Connor, A. B. et al. Abuse liability measures for use in analgesic clinical trials in patients with pain: IMMPACT recommendations. Pain 154, 2324-2334 (2013).

3. Butler, S. F., Fernandez, K., Benoit, C., Budman, S. H. \& Jamison, R. N. Validation of the revised Screener and Opioid Assessment for Patients with Pain (SOAPP-R). J. Pain 9, 360-372 (2008).

4. Chou, R. et al. Opioids for chronic noncancer pain: prediction and identification of aberrant drug-related behaviors: a review of the evidence for an American Pain Society and American Academy of Pain Medicine clinical practice guideline. J. Pain 10, 131-146 (2009). 